\title{
Secondary Network Distribution Systems Background and Issues Related to the Interconnection of Distributed Resources
}

M. Behnke, W. Erdman, and S. Horgan Distributed Utility Associates

D. Dawson, W. Feero, and F. Soudi

Consultants to Distributed Utility Associates

D. Smith

Siemens Power Technologies, Inc.

C. Whitaker

Behnke, Erdman, and Whitaker Engineering, Inc.

B. Kroposki

National Renewable Energy Laboratory 


\section{Secondary Network Distribution Systems Background and Issues Related to the Interconnection of Distributed Resources}

M. Behnke, W. Erdman, and S. Horgan Distributed Utility Associates

D. Dawson, W. Feero, and F. Soudi

Consultants to Distributed Utility Associates

D. Smith

Siemens Power Technologies, Inc.

C. Whitaker

Behnke, Erdman, and Whitaker Engineering, Inc.

B. Kroposki

National Renewable Energy Laboratory

Prepared under Subcontract No. ZAT-3-32616-03

National Renewable Energy Laboratory

1617 Cole Boulevard, Golden, Colorado 80401-3393

303-275-3000 • www.nrel.gov

Operated for the U.S. Department of Energy

Office of Energy Efficiency and Renewable Energy

by Midwest Research Institute $\bullet$ Battelle

Contract No. DE-AC36-99-G010337

Technical Report NREL/TP-560-38079

July 2005

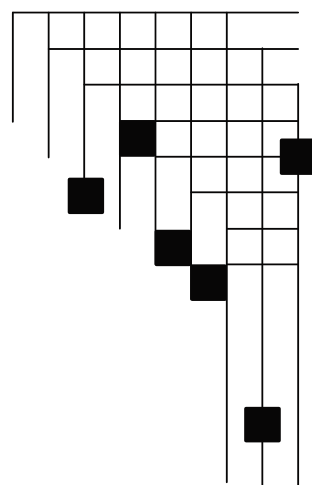




\section{NOTICE}

This report was prepared as an account of work sponsored by an agency of the United States government. Neither the United States government nor any agency thereof, nor any of their employees, makes any warranty, express or implied, or assumes any legal liability or responsibility for the accuracy, completeness, or usefulness of any information, apparatus, product, or process disclosed, or represents that its use would not infringe privately owned rights. Reference herein to any specific commercial product, process, or service by trade name, trademark, manufacturer, or otherwise does not necessarily constitute or imply its endorsement, recommendation, or favoring by the United States government or any agency thereof. The views and opinions of authors expressed herein do not necessarily state or reflect those of the United States government or any agency thereof.

Available electronically at http://www.osti.gov/bridge

Available for a processing fee to U.S. Department of Energy and its contractors, in paper, from:

U.S. Department of Energy

Office of Scientific and Technical Information

P.O. Box 62

Oak Ridge, TN 37831-0062

phone: 865.576 .8401

fax: 865.576 .5728

email: mailto:reports@adonis.osti.gov

Available for sale to the public, in paper, from:

U.S. Department of Commerce

National Technical Information Service

5285 Port Royal Road

Springfield, VA 22161

phone: 800.553.6847

fax: 703.605.6900

email: orders@ntis.fedworld.gov

online ordering: http://www.ntis.gov/ordering.htm 


\section{Contributors}

The following individuals have authored or provided comments to this document:

- Farajollah Soudi, ${ }^{*}$ consultant

- Michael Behnke, ${ }^{*}$ Distributed Utility Associates

- William Feero, ${ }^{*}$ consultant

- Douglas Dawson, ${ }^{*}$ consultant

- William Erdman, Distributed Utility Associates

- Susan Horgan, Distributed Utility Associates

- Benjamin Kroposki, National Renewable Energy Laboratory

- David Smith, Siemens Power Technologies Inc.

- Charles Whitaker, Behnke, Erdman, and Whitaker Engineering Inc.

* Primary Authors 


\section{Table of Contents}

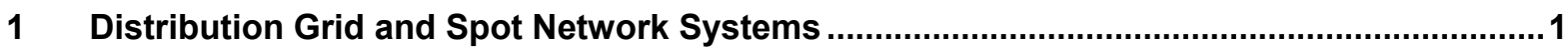

$1.1 \quad$ Scope and Purpose ....................................................................................... 1

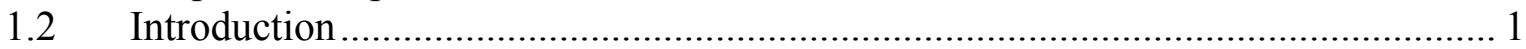

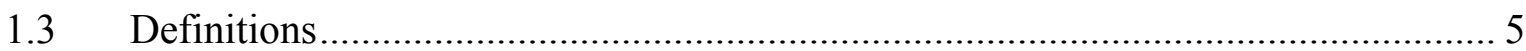

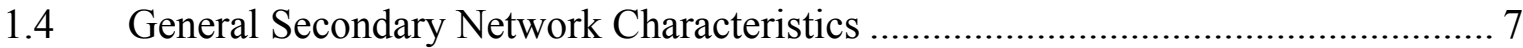

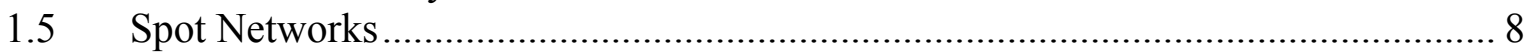

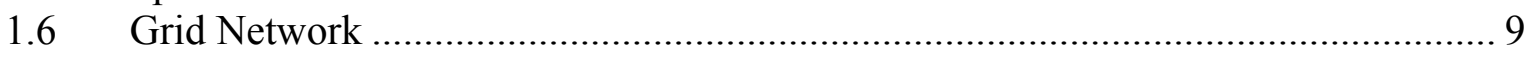

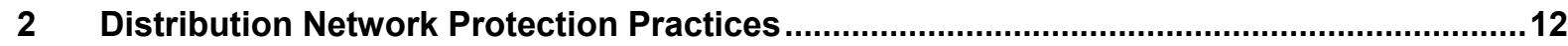

2.1 Protection of Distribution Feeders ................................................................. 12

2.2 Protection of Network Transformers .............................................................. 16

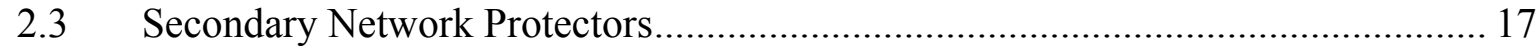

2.4 Secondary Grid Network Cable Protection....................................................... 17

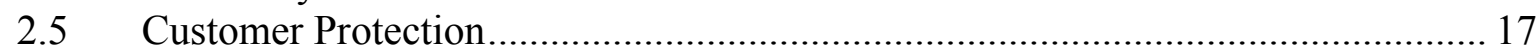

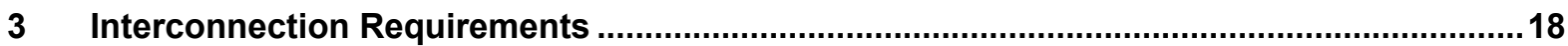

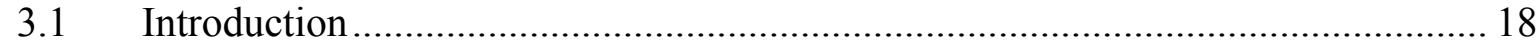

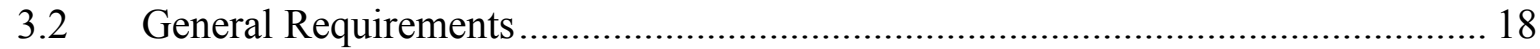

3.3 Network-Specific Interconnection Issues .................................................. 19

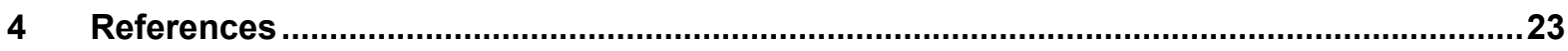

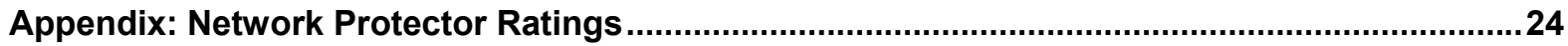




\section{List of Figures}

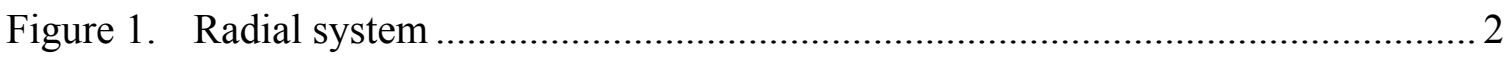

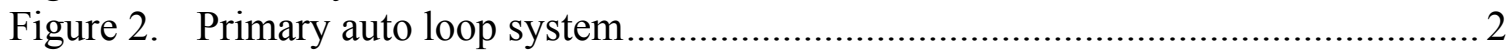

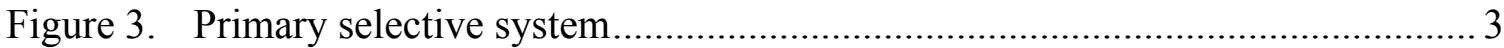

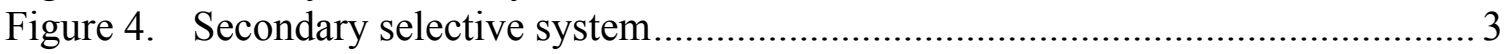

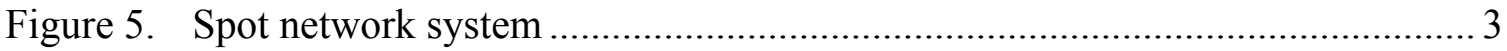

Figure 6. Secondary grid network system ......................................................... 4

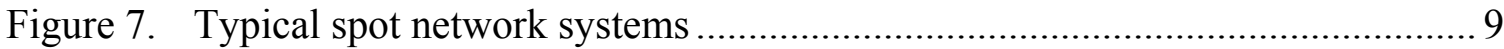

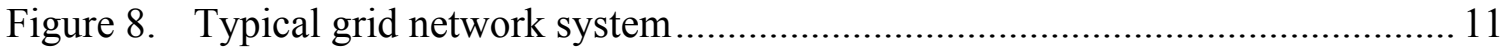

Figure 9. Master relay watt and watt-VAR trip characteristics .................................. 15 


\section{List of Tables}

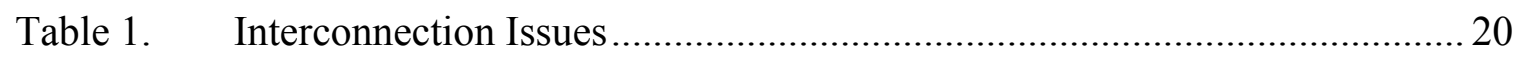

Table A-1. IEEE Network Protector Ratings at 216/125 V ………………………….... 24

Table A-2. IEEE Network Protector Ratings at 480/277 V ………………………..... 25 


\section{Distribution Grid and Spot Network Systems}

\subsection{Scope and Purpose}

This document addresses the technical considerations associated with the interconnection of distributed resources (DR) with secondary network distribution systems (or simply networks). It provides an overview of the characteristics of various distribution systems and interconnection requirements and identifies unique issues specific to network interconnections.

The purpose of this document is to identify the network-specific interconnection issues for which test protocols should be developed and to assist in test facility design and test plan development. Recommended criteria and requirements for the interconnection of DR with network distribution systems are presented.

\subsection{Introduction}

To clarify the unique characteristics of grid and spot network service, a brief review of common modes for distributing electric power is presented.

Utilities use distribution systems to serve their customers with reliable quality power. The most common distribution system is a simple radial circuit that can be $100 \%$ overhead, $100 \%$ underground, or a combination of both. The most common distribution feeder characteristics and classifications are listed below.

1. The distribution voltage classes for most utilities are $5 \mathrm{kV}, 15 \mathrm{kV}, 25 \mathrm{kV}$, and $35 \mathrm{kV}$.

2. Radial distribution lines can be less than a mile to more than 20 miles long. This distance is from the substation to the furthest service point; it is not the total mileage of all branches.

3. A distribution line load can be as high as $1,200 \mathrm{~A}$, but the range of $300-400 \mathrm{~A}$ is common.

4. The short-circuit duty at each distribution substation varies depending on transformer size and voltage class, ranging from $10 \mathrm{kA}$ to $50 \mathrm{kA}$.

5. Distribution feeders include control devices. The most common are shunt capacitors to meet local volt-ampere reactive (VAR) requirements or support voltage regulation. Voltage boosters or voltage regulators are used to maintain adequate line voltage. Series reactors can be employed to limit the fault current. Two winding or autotransformers may be used on the feeder to change the distribution voltage class.

6. Multi-grounded, uni-grounded, ungrounded, and resistively or reactively grounded distribution systems are used in the industry. The multi-grounded four-wire distribution system and uni-grounded three-wire distribution systems are most common in North America. 
7. Protective devices are installed on distribution feeders to mitigate safety hazards to the public, prevent or minimize damage to equipment, and improve service reliability by clearing an abnormal condition by removing a small section of the circuit for a given fault. Distribution feeder protection consists of a circuit breaker at the substation and line reclosers, sectionalizers, interrupters, and fuses at intermediate locations along the main feeders and laterals.

The protection devices and philosophy described above do not produce perfect results. Utility attempts to improve reliability are generally focused on two methods:

1. Preventive and corrective maintenance

2. Backup capability at either the primary or secondary voltage levels through manual switching, automatic switching, or network service.

Examples of distribution electrical service are shown in figures 1-6. Switching schemes for improved reliability include a primary auto loop scheme, a primary selective scheme, and a secondary selective scheme. The primary auto loop scheme is applied in radial distribution circuits. The primary selective scheme is applied in concentrated load areas, and the secondary selective scheme is applied in industrial plants and institutions.

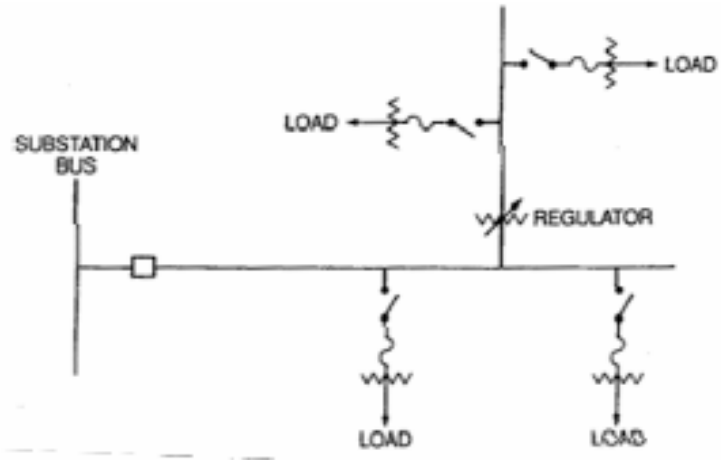

Figure 1. Radial system

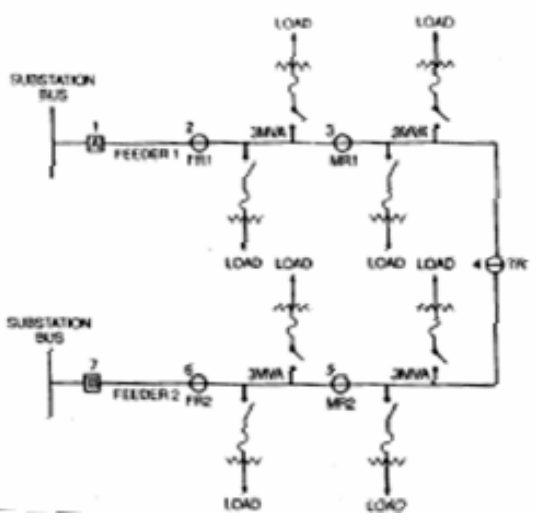

Figure 2. Primary auto loop system 


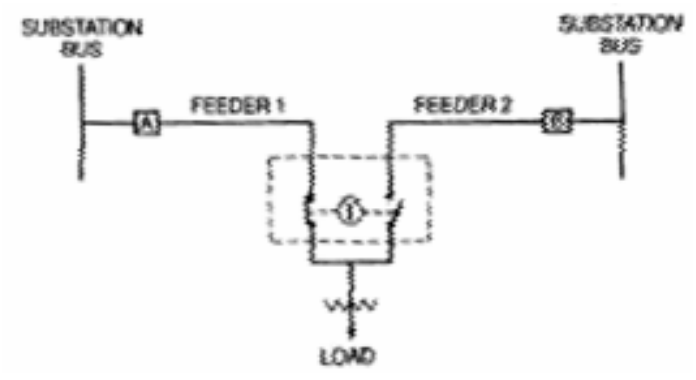

Figure 3. Primary selective system

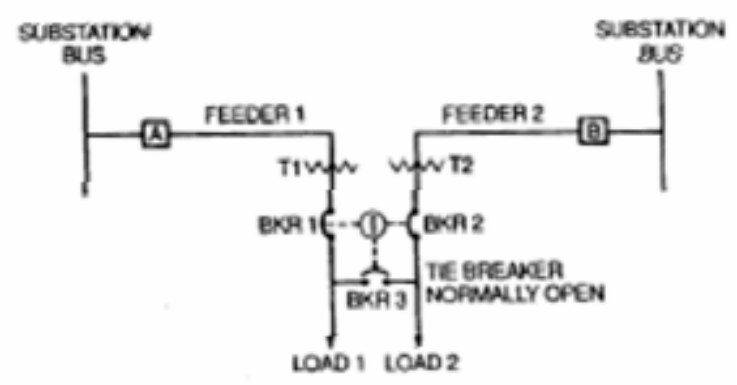

Figure 4. Secondary selective system

To provide maximum reliability and operating flexibility, utilities use spot and grid network systems in congested area such as metropolitan and suburban business districts.

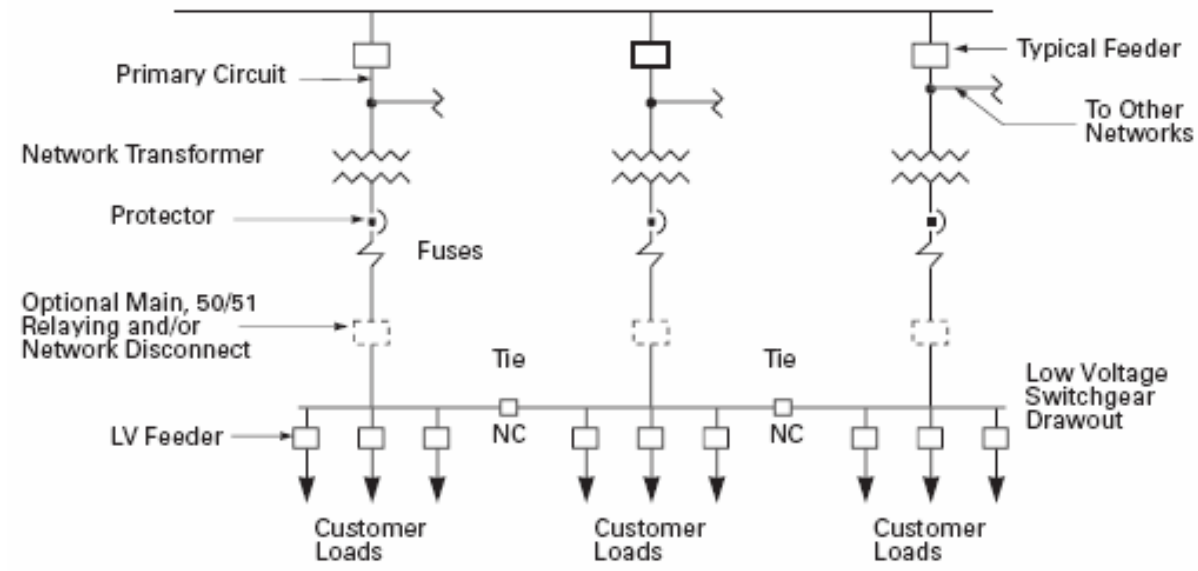

Figure 5. Spot network system 


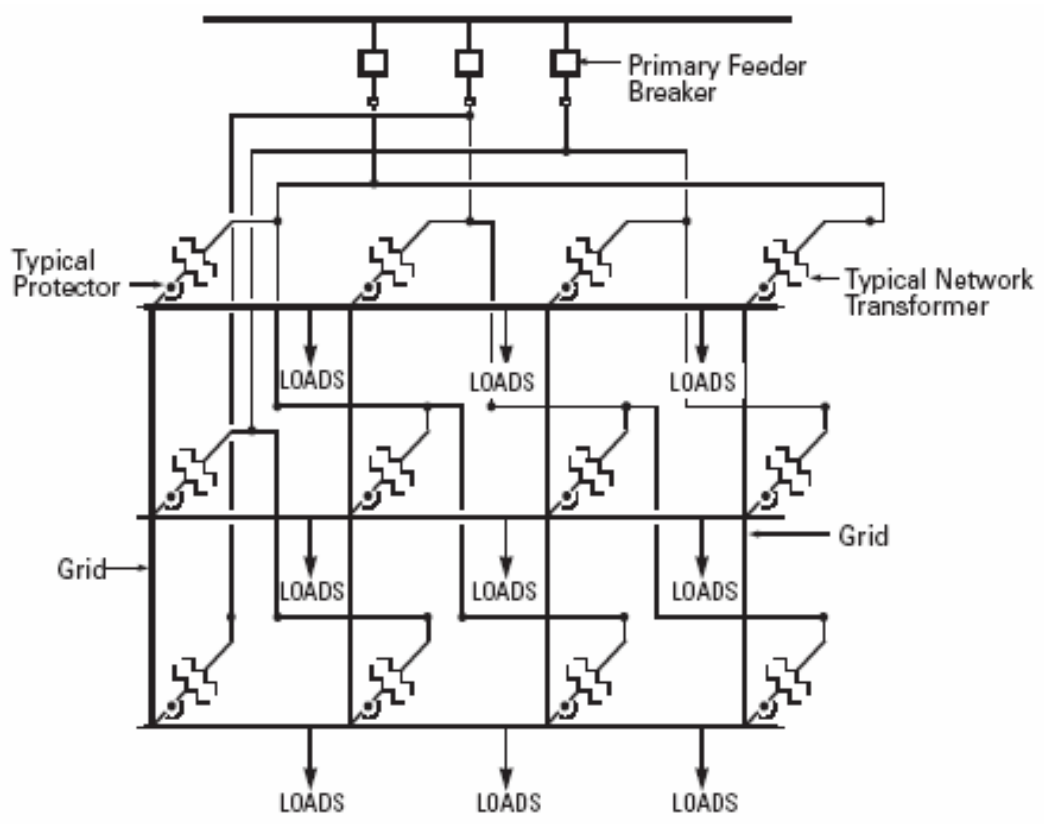

Figure 6. Secondary grid network system (one primary source shown; may have two or more) 


\subsection{Definitions}

Consistent definitions are critical to understanding the design and operation of secondary network distribution systems. Alternate definitions for some of the terms may be found in different regions. These definitions represent the most common usage.

Cable limiter: An enclosed fuse for disconnecting a faulted cable from a secondary network distribution system and protecting the unfaulted portion of that cable from serious thermal damage.

Cycling: The undesirable cyclical tripping and closing of a network protector because of external (load) conditions.

Left unchecked, cycling may eventually lead to failure of the network protector.

Grid network: A secondary network system with geographically separated network units and the network-side terminals of the network protectors interconnected by low-voltage cables that span the distance between sites.

The low-voltage cable circuits of the grid networks are typically highly meshed and supplied by numerous network units. This is also referred to as an "area network" or "street network."

Network master relay: An electromechanical poly-phase relay with two functions: (1) opening the network protector when power flow is from the low-voltage side to the high-voltage side of the network transformer and (2) closing the network protector in conjunction with the electromechanical network-phasing relay when transformer voltage is higher than network voltage and leads the network in phase angle.

Network protector fuse: A backup protective device for the network protector.

Network protector: An assembly composed of a circuit breaker and its complete control equipment for: (1) automatically disconnecting a transformer from a secondary network distribution system in response to predetermined electrical conditions on the primary feeder or transformer and (2) connecting a transformer to a secondary network through manual or automatic control responsive to predetermined electrical conditions on the feeder and the secondary network distribution system.

The network protector is usually arranged to automatically connect its associated transformer to the secondary network distribution system when conditions are such that the transformer, when connected, will supply power to the secondary network distribution system and to automatically disconnect the transformer from the network when power flows from the secondary network distribution system to the transformer (from IEEE C57.12.44-2000).

Network system: A collection of spot networks, secondary grid networks, or combinations of such networks and the primary feeders that supply them. 
Network transformer: A transformer designed for use in a vault to feed a variable capacity system of interconnected secondaries. A network transformer may be submersible or vault. It usually, but not always, has a provision for attaching a network protector (from IEEE C57.12.80-1978). Dry transformers are also used for spot network applications.

Network unit: A network unit consists of a primary disconnect and grounding switch, a network transformer, and a network protector.

Primary network feeder: A feeder that supplies energy to a secondary network distribution system or the combination of a secondary network distribution system and other radial loads.

Dedicated primary network feeders are feeders that supply only network transformers for the grid network, the spot network, or both. Non-dedicated primary network feeders, sometimes called combination feeders, are feeders that supply both network transformers and non-network load.

Pumping: The rapid, uncontrolled, unintentional, and intolerable repetitive tripping and closing cycle of a network protector, normally because of a failure in the network protector control circuitry.

If not detected and corrected, pumping will quickly lead to failure of the network protector.

Secondary network: The low-voltage circuits supplied by the network units (the network transformer and its associated network protector). Unless specifically excepted, in this document, any reference to "the network" means the secondary network.

Secondary network distribution system ("network"): An AC power distribution system in which customers are served from three-phase, four-wire low-voltage circuits supplied by two or more network transformers whose low-voltage terminals are connected to the low-voltage circuits through network protectors. The secondary network system has two or more highvoltage primary feeders, with each primary feeder typically supplying 1-30 network transformers, depending on network size and design. The system includes automatic protective devices intended to isolate faulted primary feeders, network transformers, or low-voltage cable sections while maintaining service to the customers served from the low-voltage circuits.

Unless otherwise stated, in this document, "network" means the secondary network distribution system.

Spot network: A secondary network distribution system that consists of two or more network units at a single site. The secondary network-side terminals of these network units are connected together with bus or cable. The resulting interconnection structure is commonly referred to as the paralleling bus or collector bus. In spot networks, the paralleling bus does not have low-voltage ties to adjacent or nearby networks. Such spot networks are sometimes called isolated spot networks to emphasize that there are no secondary voltage connections to network units at other sites. 
Spot network with reach: A spot network with a secondary voltage cable connection to one or more neighboring spot networks or to a nearby grid network. These reach connections are usually of a capacity limited to the rating of one of the network units supplying either spot network.

Underground connectors: Underground connectors in manholes and transformer vaults that provide for multiple connections at a single junction point.

\subsection{General Secondary Network Characteristics}

Although networks are classified as either spot or grid, all networks share certain characteristics. These characteristics are described below.

1. Network systems are designed based on redundant facilities. Any single equipment failure will not result in service outage on the network.

2. Each network is served by at least two primary feeders.

3. A primary feeder may serve a single network unit or many network units at different sites and may also serve radial distribution loads.

4. The primary feeders for a network system are generally served from a single substation but may be served by different substations. When supplied from different substations, phase angle difference and voltage magnitude difference must be minimized if acceptable operation is to be obtained.

5. The bus configuration at the distribution feeder varies. The configuration can be a single breaker, double breaker with both breakers closed, or double breaker with one breaker open and with a transfer switch scheme or sectionalizing bus tie breaker. Utilities also use ring bus arrangements, double synchronizing bus designs, and others such that a bus fault in the station will not result in an outage to more than one primary network feeder.

6. A network unit consists of a high-side disconnect or grounding switch, a network transformer, and a network protector (with master relay, phasing relay, and fuses).

7. The primary network voltage classes range from $5 \mathrm{kV}$ to $35 \mathrm{kV}$.

8. Typical network transformer sizes are $300 ; 500 ; 750 ; 1,000 ; 1,500 ; 2,000 ;$ and 2,500 kVA. Transformers with $208 \mathrm{Y} / 120 \mathrm{~V}$ secondaries do not exceed 1,000 kVA in rating.

9. The transformer impedance is specified in ANSI C57.12.40-2000 and ranges $4 \%-7 \%$.

10. The primary feeder can be either a three-wire or four-wire system.

11. The transformer connections are commonly delta primary-wye grounded secondary for three-wire feeders and wye grounded-wye grounded for four-wire feeders.

12. Protector ratings are given by IEEE Standard C57.12.44 (see appendix). They can be in non-submersible housings, submersible housings, or housings suitable for mounting within a low-voltage switchgear assembly. The ratings of the protectors vary depending on the manufacturer, the type of the protector, and the secondary voltage. Continuous current ratings are 800-6,200 A, 216-600 V. The interrupting ratings are 30,000-85,000 $\mathrm{A}$, and close and latch ratings are 25,000-65,000 A. 
13. Network protectors are maximum current-rated devices and have no published overload rating. Protector ratings are generally $33 \%-67 \%$ higher than the continuous ratings of the transformers they protect to take advantage of short-term transformer overload capability.

\subsection{Spot Networks}

Spot network systems are designed to provide highly reliable $277 / 480-\mathrm{V}$ or $120 / 208-\mathrm{V}$ service to a single site. (In rare cases, $240-\mathrm{V}$ delta ungrounded service may be provided.) These systems are commonly applied in high load-density areas such as metropolitan and suburban business districts. Figure 7 shows two typical spot network systems, each with two network units.

Spot networks have the following characteristics:

1. Most utilities operate each network system isolated, but some utilities may provide alternative sources to the primary network feeders.

2. A spot network consists of two or more network transformers (a three-transformer spot network is common) that are paralleled at the secondary bus.

3. In some cases, fast-acting secondary bus tie breakers (not present in Figure 7 but shown in Figure 5) may be applied between bus sections to isolate faults in the secondary switchgear and limit loss of service to those loads connected to the faulted equipment.

In the spot networks of most utilities, a fault or failure of the paralleling bus (collector bus) will result in a service outage. This is one part of the system for which redundancy does not exist for most utilities, so its design and integrity is of great importance.

Also, for some utilities, a fault on the medium voltage bus in the substation that supplies the primary feeders will result in an outage to the network. This does not apply to those utilities that have ring bus designs, double synchronization bus designs, etc., for their medium-voltage substations. 


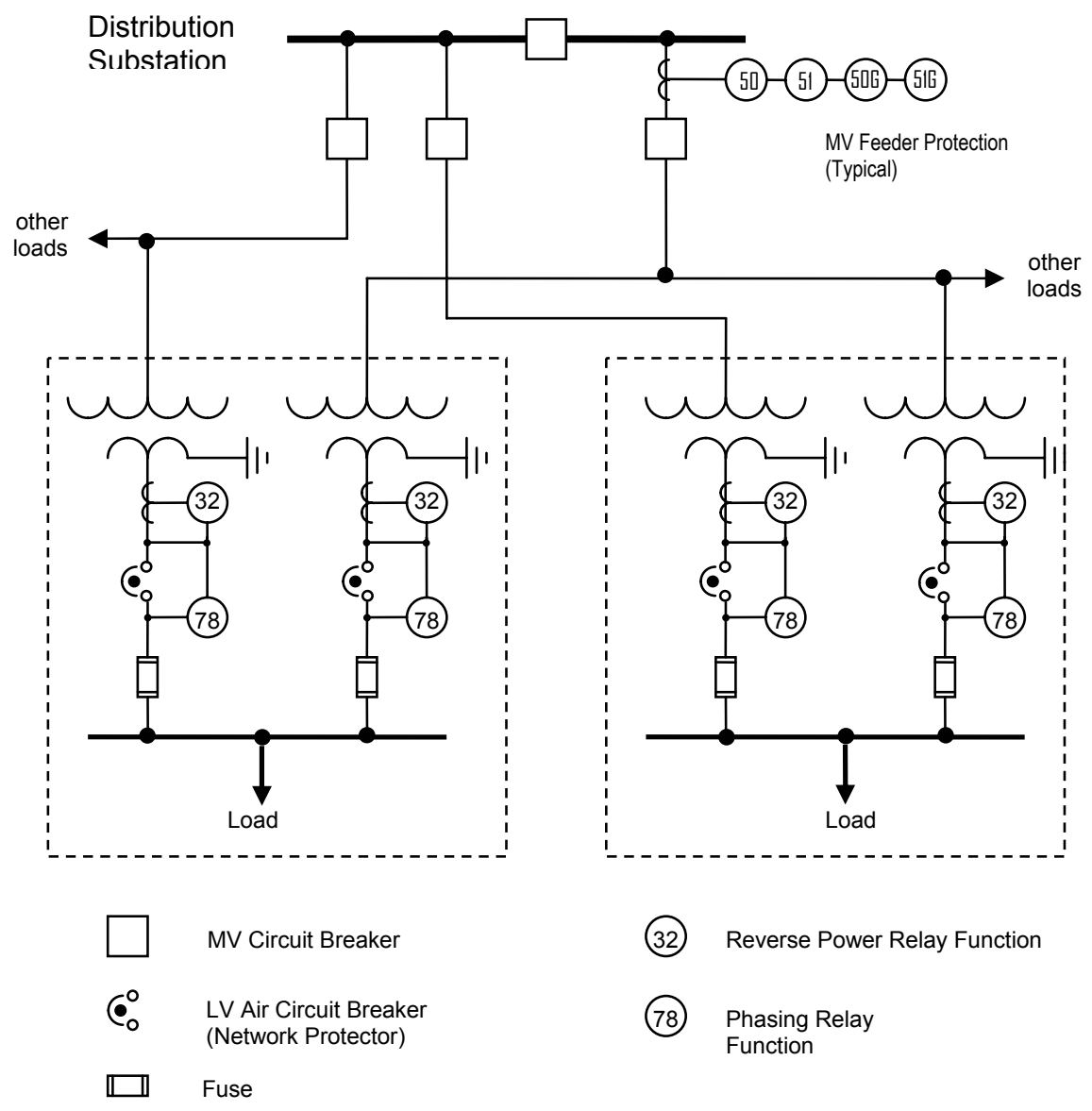

Figure 7. Typical spot network systems

\subsection{Grid Network}

The "grid" secondary network distribution system consists of an interconnected grid of circuits operating at utilization voltage and energized from a number of primary feeder circuits and network units. The number of cables that tie the secondary buses to one another can be anywhere from one to dozens. These cables are also referred to as secondary mains. The numerous cables allow for multiple current paths from every network unit to every load within the grid.

Cable limiters protect some of these cables (by "limiting" thermal damage to the cables under fault conditions). Figure 8 shows a portion of a typical grid network system fed from a single substation. The grid or mesh is designed so adjacent network transformers are served by different primary feeders. 
Grid networks have the following characteristics:

1. The secondary voltages are either $208 \mathrm{Y} / 120 \mathrm{~V}$ or, in rare cases, $480 \mathrm{vY} / 277 \mathrm{~V}$.

2. The integrity of the grid network is based on multiple paths through individual cables. This integrity is maintained by individual cables, and, if used, cable limiters burning clear any faulted cable sections.

3. The conductors from which customer service is tapped generally follow the geographical pattern of the load area and are located under streets and alleys.

4. Load flow within the grid network will significantly change as a function of:

- Medium-voltage ${ }^{1}$ feeder outage conditions

- Changing customer load conditions

- Reduced current carrying capacity because of cleared cable limiters.

Primary feeder outages and burned-off cables or cleared limiters because of previous faults within the grid will cause changes in load flow that are not readily detected. The inherent system redundancy generally prevents any customer from experiencing poor power quality. Load flow analysis is necessary to understand the maximum current levels at any point and may be required to determine which network units are exposed to cycling under minimum load conditions.

\footnotetext{
${ }^{1}$ Throughout this document, low voltage is less than $600 \mathrm{~V}$, and medium voltage is roughly $600 \mathrm{~V}-40 \mathrm{kV}$.
} 


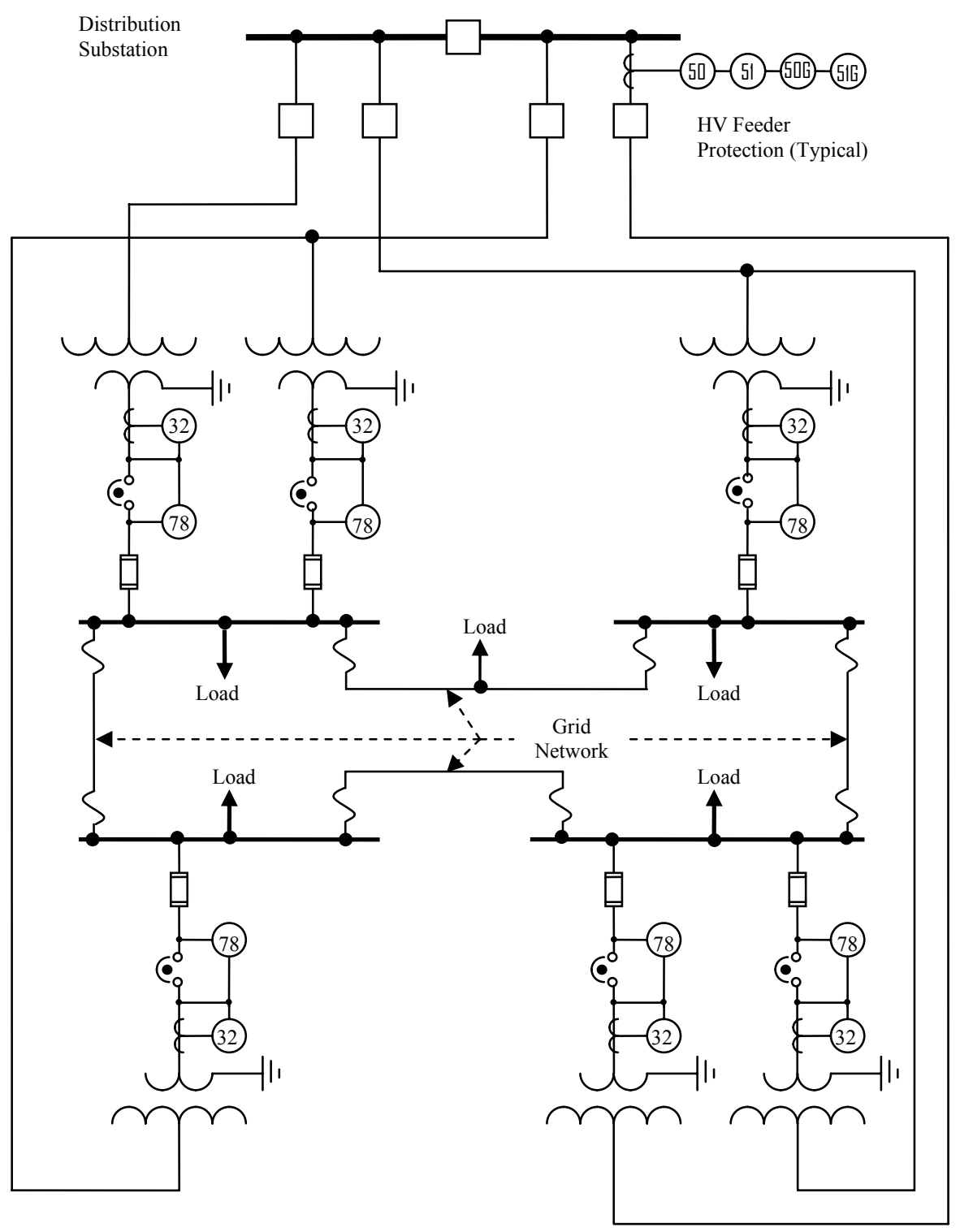

Figure 8. Typical grid network system 


\section{Distribution Network Protection Practices}

\subsection{Protection of Distribution Feeders}

De-energizing a faulted feeder in an ordinary radial distribution system requires only a single tripping action: opening the feeder's medium-voltage circuit breaker (MVCB) at the supplying distribution substation - assuming there is no DR on the feeder. In contrast, de-energizing a faulted distribution feeder that supplies a spot or grid secondary network system requires at least two (and usually more) tripping operations:

1. Open the feeder's MVCB.

2. Open all the secondary network protector circuit breakers at the network transformers supplied from that feeder.

As shown in figures 7 and 8, the protective relaying for the MVCB of network feeders is typically conventional phase and ground overcurrent protection, similar to that used on radial feeders.

The network protectors are opened by the Device 32 reverse-power function ${ }^{2}$ at each network transformer. These operations may be sequential (i.e., some or all of the network protectors may not open until the MVCB has opened).

The reverse-power function operates only for real power (watt) flow in the reverse direction (i.e., flow from the low-voltage network toward the transformer). Under non-fault conditions, power flow is normally from the transformer to the network, so the relay does not trip. When a primary feeder is faulted, the reverse-power function trips from one or more of the following:

1. Real power flow from the network to the feeder fault

2. Real power flow from the network to other loads or network units on the feeder

3. Real power flow from the network to supply the core losses of the network transformer.

Power flow to the fault (1) is affected by fault type, network transformer primary winding connection (delta or wye), and whether the feeder MVCB has opened. Because the master relays are poly-phase devices responding to the net effect of power flow on all three phases, unbalanced feeder faults in the presence of load power flow may not result in a net reverse power flow through the relay.

\footnotetext{
${ }^{2}$ In network units that use electromechanical relays, reverse power is one of the two functions incorporated in the network protection relay termed the "master relay." The other function of the master relay is to set the minimum voltage difference that must be present to permit reclosing of the network protector. In solid-state and microprocessor-based network protector relays, all the required protection functions are incorporated in a single relay.
} 
A case of particular importance is a single-line-to-ground fault on the primary feeder with a network transformer primary winding connection of delta. In this case, once the feeder MVCB has opened, the "fault" current is limited to a value permitted by the primary system capacitance, which leads to small currents and very little power flow. This case requires great care in design to ensure that a possibly hazardous condition is cleared by the network protector control devices.

Reverse power flow to other feeder loads (2) generally occurs only after the feeder MVCB has opened. Its magnitude depends on whether the primary feeder supplies radial loads in addition to network loads. If the feeder has only network loads, then it is possible for the magnitude of (2) to be zero.

Because the effects of (1) and (2) may be negligible in some cases, network protection design usually relies on (3) to ensure all network protectors open for an outage of the primary feeder. Transformer core power losses are very small-typically $0.1 \%-1 \%$ of the rating of the transformer. Consequently, the reverse power pickup settings of the master relays are very sensitive, on the order of $0.1 \%-0.5 \%$ of the rated power of the network transformer. The very sensitive reverse-power pickup of the network relays is a major concern in the application of DRs on secondary networks. The slightest power export from the network, even lasting just 3 cycles, may open all the network protectors serving the facility and thereby create an island.

\subsubsection{Modified Tripping Characteristics}

Some network applications require modification of the basic instantaneous reverse-power protection to optimize performance for specific conditions. Microprocessor-based network relays incorporate these features as optional settings. Modifications to electromechanical network relays generally require additional devices.

\section{Time-Delayed Trip}

Large regenerative loads, such as elevators, may substantially reduce the network real power load for many seconds. In applications in which such loads can cause a temporary reversal of power through the network unit, it may be desirable to delay the reverse-power trip function to avoid unnecessary operations of the network protector circuit breaker. Because the time delay is required only for moderate amounts of reverse power (less or somewhat greater than the rating of the network unit), time-delayed trip incorporates a current-supervision function that bypasses the time delay when the network unit current is higher than the expected level of reverse-power flow. The net effect is a two-level reverse-power function: time-delayed for low levels of reverse power but instantaneous at higher levels, as occur during faults on the primary feeder. ${ }^{3}$

For applications of DRs on secondary network systems, the time-delayed trip modification may be useful for preventing unwanted network protector trips that would otherwise be caused by power or fault current flow from the DRs.

\footnotetext{
${ }^{3}$ In units with electromechanical network relays, this time delay with current supervision function was performed by an auxiliary relay called the "BN" relay. As a result, the feature is often referred to as the "BN" function in microprocessor-based network relays.
} 


\section{Watt/VAR Sensing}

The basic reverse-power detection function of the network relays may not always be adequate for detecting all types of primary feeder faults. Specifically, in a system with single-phase protection devices (fuses) in the primary feeder, or as protection for the network transformer, a single phase-to-ground fault may result in blowing only one fuse in the high-voltage feeder. In such a case, real power continues to flow toward the network on the two unfaulted phases while fault current (mostly VARs) flows from the network toward the primary fault.

Because the reverse-power function reacts to the net power flow of all three phases, it may not be able to detect such a condition. Watt/VAR sensing modifies the characteristic of the reversepower function, as shown in Figure 9, so that it responds to reverse VARs as well as reverse watts, which enables the network relay to detect the abnormal supply condition and open the network protector.

Because watt/VAR sensing modifies the relay characteristic to allow tripping on reverse VAR flow, it creates the possibility of unwanted tripping for leading power factor load and prevents tripping during a capacitive backfeed to the primary feeder. To avoid this possibility, watt/VAR sensing is usually controlled by the same current supervision function used for time-delayed trips. Thus, pure reverse-power detection is employed at low levels of current, and watt/VAR sensing is switched in at higher levels. ${ }^{4}$

\footnotetext{
${ }^{4}$ In one microprocessor-based network relay, the shift from watt trip to watt/VAR trip characteristic was based on the magnitude of the negative-sequence voltage.
} 


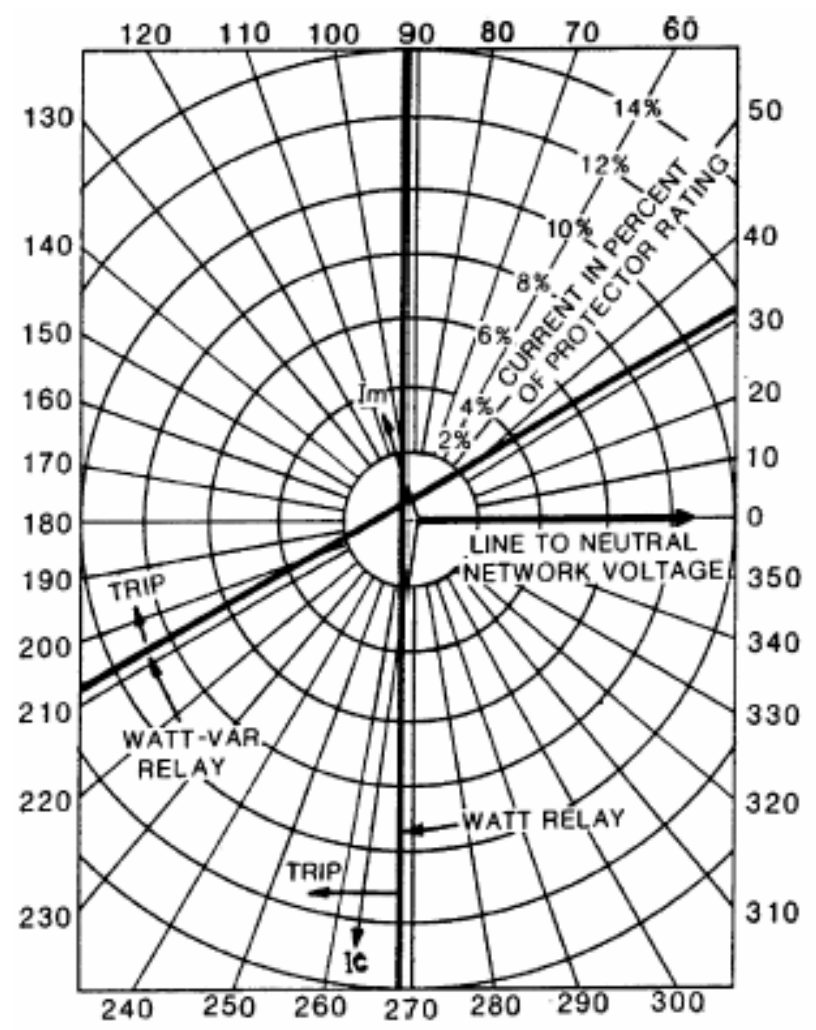

Figure 9. Master relay watt and watt-VAR trip characteristics

\subsubsection{Restoration}

Once the faulted primary feeder has been repaired and re-energized, the network transformer units are expected to automatically reclose their network protector circuit breakers, which restores their supply to the network, provided the ensuing watt and VAR flows will be into the network. This reclosing is controlled by the master relay function and phasing relay function. ${ }^{5}$ Reclosing supervision by these relays ensures that the network protector will not immediately re-open from reverse power flow.

Installing a DR on a secondary network system may interfere with this reclosing plan by offsetting the network load to a point at which an open network protector cannot reclose because the network voltage or phase angle is outside the permissive closing boundary. Modifying the relay reclosing settings to accommodate the DR may lead to more frequent network protector openings under light load conditions.

\footnotetext{
${ }^{5}$ In network systems that use electromechanical relays, separate "master" and "phasing" relays operate together to supervise reclosing. The master relay sets the minimum voltage by which the network transformer secondary voltage must exceed the network voltage to permit reclosing. The phasing relay sets the angle by which the transformer secondary voltage must lead the network voltage to ensure that real power will flow from the transformer to the network when the network protector is closed. Microprocessor-based network relays combine these functions, plus the reverse-power protection, in a single relay.
} 
Network phasing relays are designed to measure phase angle under the static conditions that normally occur in network systems (i.e., the phase angle is either slightly leading or lagging). They are not equipped to deal with the rotating phase-angle conditions that might occur if the network is supplied from an isolated DR while the network units are attempting to reclose. If such a condition were to occur, the network protector breakers would likely "pump" (i.e., repeatedly open and close), paralleling and separating the two systems, until the network protector fails. It is also possible that the network protector would close out of phase and damage the DR, the network protector, or both. Network relays were never intended to control closing of the network protector when the systems on either side of the protector are not synchronized.

\subsection{Protection of Network Transformers}

Protection practice for network transformers differs from that of conventional distribution transformers in two important ways:

1. Medium-voltage transformer fuses are often not used. Because service to the network loads is not interrupted, it is deemed acceptable to de-energize the entire primary feeder for a network transformer fault.

2. It is necessary to open the low-voltage connection to the transformer as well as the high-voltage connection because the network is a source of supply to the faulted transformer.

Faults in the medium-voltage cable connections to the transformer, disconnect and grounding switch, or tap changer or in the medium-voltage winding are similar in magnitude to the feeder faults discussed previously and are cleared similarly. Faults in the transformer's low-voltage winding are isolated from the network by opening the network protector breaker but have longer clearing times from the high-voltage side because the impedance of the transformer limits the fault current seen by the feeder overcurrent relays. Further, ground faults in the lowvoltage winding, or the low-voltage leads, are not detected by the feeder ground relays if the transformer high-voltage winding is delta-connected. In some cases, the feeder relays may not clear a low-voltage fault until sufficient transformer damage occurs to involve the high-voltage winding. ${ }^{6}$

Fuses in the low-voltage leads of the network protector provide backup protection for the network protector for faults on the primary feeder or in the network transformer and isolate the network unit from the network for low-voltage faults between the network relay current transformers and the fuses. To ensure selective clearing of such faults, cable limiters and the secondary fuses of adjacent network transformers must coordinate with the secondary fuse of the faulted network unit. Such coordination is aided by the multiplicity of fault current sources and the single fuse in the path of the total fault current. However, such coordination is not possible in the case of a twounit spot network.

\footnotetext{
${ }^{6}$ ANSI/IEEE Std. C37.108, IEEE Guide for the Protection of Network Transformers, discusses this problem in detail and provides suggestions for improving the capability to detect and clear network transformer faults.
} 


\subsection{Secondary Network Protectors}

The network protector consists of a special air power breaker, a breaker operating mechanism, a network relay, and control equipment. Units are available in semi-rustproof and submersible enclosures for separate or transformer throat mounting. Switchgear mounting is also possible.

Design and operational descriptions for these devices can be obtained from manufacturers of the protectors (Cutler-Hammer and Richards Manufacturing Co.). It should be noted that the interrupting capability of the network protector breakers is designed for the fault current levels (magnitude and X/R ratio) ordinarily encountered in low-voltage network systems. Network protectors are typically designed for $\mathrm{X} / \mathrm{R}$ ratios in the range of 6-8, whereas breakers are designed for $\mathrm{X} / \mathrm{R}$ ratios greater than 20 . Concerns have been raised about the ability of the network protectors to successfully interrupt fault currents in systems in which DRs significantly change fault current magnitudes or $\mathrm{X} / \mathrm{R}$ ratios, though network protectors are currently operating on network systems with $\mathrm{X} / \mathrm{R}$ ratios above 8 .

\subsection{Secondary Grid Network Cable Protection}

Underground cables connect secondary buses, moles, and crabs in the grid network system to one another. The number of cables per phase and cable size depend on the maximum possible load flow. The number of cables can range from one to dozens per phase.

Cable-to-cable limiters protect these cables. Limiters are designed for 4/0-, 250-, 300-, 350-, 400-, 500-, and 750-kcmil cables. Limiters are also available for connecting cable to bus and cable to moles. Limiters are also included in "fusible crabs." These limiters should coordinate with the network protector fuses and the insulation damage characteristics of the cable.

Some utilities do not use limiters for cables $4 / 0$ and smaller because they assume that cables are small enough to burn clear.

\subsection{Customer Protection}

The protection device at the customer service point is usually a main breaker, but in old facilities, fuses might be used instead of a main breaker. It is important that this device be properly coordinated with each of the network protector fuses and secondary tie breakers (if present). 


\section{Interconnection Requirements}

\subsection{Introduction}

The increasing demand for the interconnection of DRs with utility distribution systems has led utilities to establish their own interconnection procedures and requirements. Although each utility has created interconnection requirements based on its own power system, to a large degree, general requirements are common throughout the industry.

In California, interconnection requirements among the three investor-owned utilities have been harmonized through the California Public Utilities Code Electric Rule 21. Rule 21 itself has been harmonized with the IEEE 1547-2003 interconnection standard. These standards specify that DRs must be equipped with protection devices that can detect electrical faults in the system and immediately remove the appropriate unit(s) from the utility network to eliminate their contribution of fault current into the utility system. Other general requirements include manual load break disconnect devices, dedicated transformers, and islanding detection. The protection requirements for the DR facilities are based on their output capability, type of generator (e.g., synchronous, induction, or DC inverter), and the point of interconnection.

\subsection{General Requirements}

IEEE 1547 provides a uniform standard for the interconnection of DRs with electric power systems. The standard provides technical specifications and requirements in the following areas:

- 4.1 - General requirements

- 4.2 - Response to area electric power system abnormal conditions

- 4.3 - Power quality

- 4.4 - Islanding.

Network interconnection is addressed under general requirements. This section provides guidance for spot networks and leaves grid networks "under consideration for future revisions." Some have complained that the specifications for spot networks are worded in such a way that nearly all spot network interconnections will be subject to a site-specific engineering review to determine actual requirements. (IEEE 1547 acknowledges that systems larger than 5\% of the spot network's maximum load may require study to determine if the requirements can be met.) Many state and local jurisdictions are attempting to develop interconnection requirements for network systems using IEEE 1547 guidance. 
Network service is a premium service. Its higher reliability comes at a higher price than service from the more common radial distribution system. Operation, maintenance, and design tend to be assigned to specialists within a given utility. These conditions, along with generally higher fault levels, the age and sophistication (or lack thereof) of most network hardware, and the lack of experience by utility people in the connection of major DR sources have, so far, limited the development of comprehensive interconnection requirements for low-voltage network systems.

A thorough discussion of problems and solutions is necessary to avoid degrading the advantages of network service. In the following section, such issues are presented with the goal of identifying engineering solutions or defining tests to support solutions.

\subsection{Network-Specific Interconnection Issues}

Unique problems are associated with the interconnection of DR with network distribution systems. The issues vary depending on whether the interconnection is made radially on a primary feeder that supplies a network, on a spot network secondary, or on a grid network secondary.

Table 1 was prepared for a network experts meeting held in January 2005 as a way to organize the discussion of issues related to the interconnection of DRs in networks. 
Table 1. Interconnection Issues

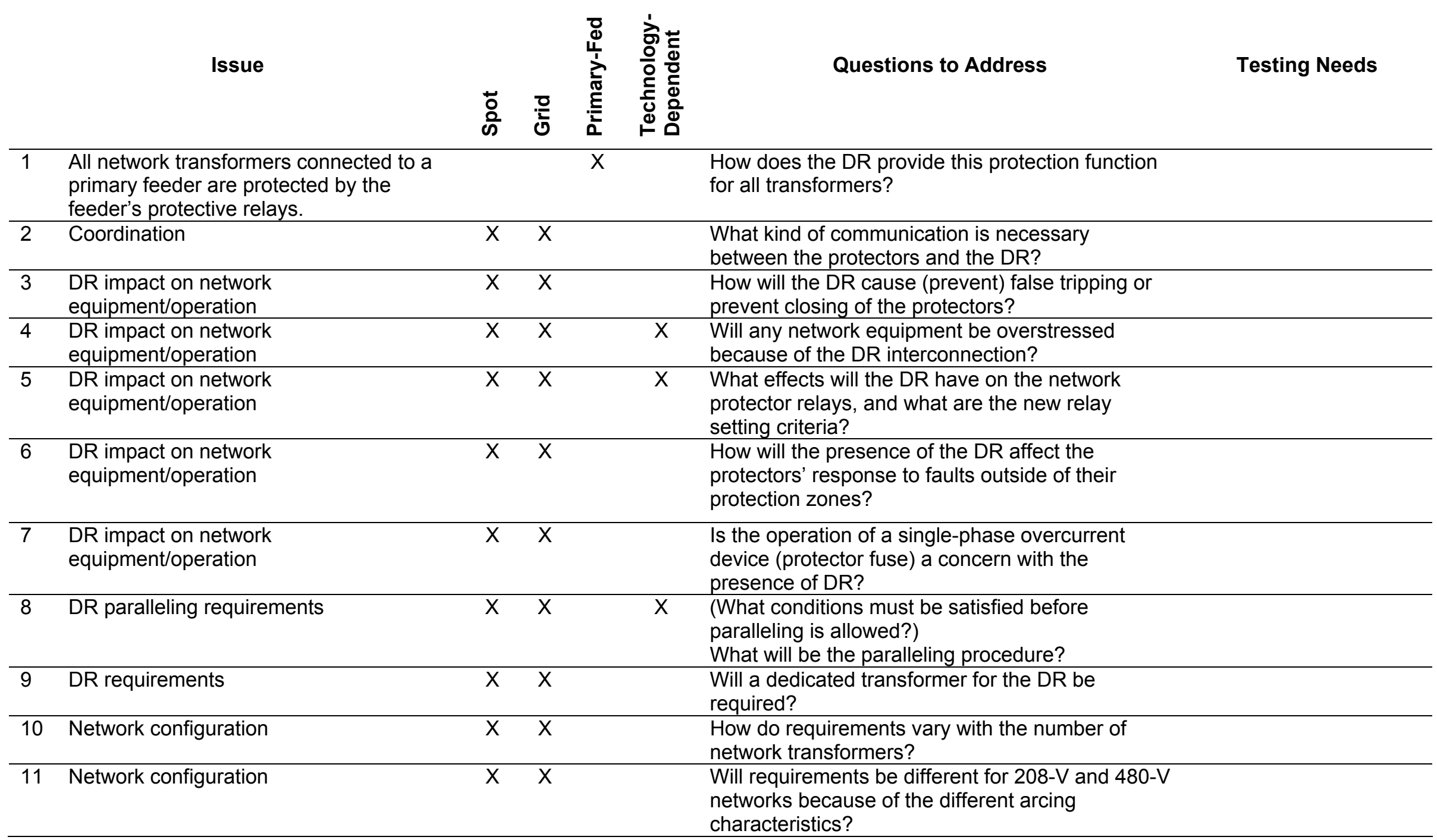




\begin{tabular}{|c|c|c|c|c|c|c|c|}
\hline & Issue & $\begin{array}{l}\ddot{\circ} \\
\ddot{n}\end{array}$ & 은 & 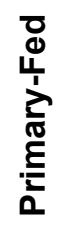 & 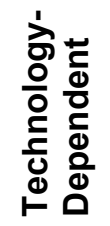 & Questions to Address & Testing Needs \\
\hline 12 & Network configuration & & $\mathrm{X}$ & & & $\begin{array}{l}\text { Will the presence, or lack, of cable limiters on the } \\
\text { secondary cables result in different DR } \\
\text { interconnection requirements? }\end{array}$ & \\
\hline 13 & Network configuration & & $\mathrm{X}$ & & $\mathrm{X}$ & $\begin{array}{l}\text { Will the presence of dozens to hundreds of } \\
\text { network transformers spread out over a wide area } \\
\text { result in different requirements? }\end{array}$ & \\
\hline 14 & Network configuration & & $\mathrm{X}$ & & $\mathrm{X}$ & $\begin{array}{l}\text { Will changes in power flow over the daily or } \\
\text { weekly load cycle result in protector cycling at a } \\
\text { point remote from the DR's point of common } \\
\text { connection? }\end{array}$ & \\
\hline 15 & Network line configuration & $\mathrm{X}$ & $\mathrm{X}$ & & & $\begin{array}{l}\text { Will different protection requirements apply to } \\
\text { network systems supplied from three-wire and } \\
\text { four-wire primaries? With delta-wye or wye-wye } \\
\text { transformers? }\end{array}$ & \\
\hline 16 & $\begin{array}{l}\text { Protector breakers are not designed to } \\
\text { interrupt fault current from generators or } \\
\text { withstand out-of-phase conditions across } \\
\text { the open switch. }\end{array}$ & $\mathrm{X}$ & $\mathrm{X}$ & $\mathrm{X}$ & $\mathrm{X}$ & $\begin{array}{l}\text { How will the protector be prevented from isolating } \\
\text { DRs from the utility system? }\end{array}$ & \\
\hline 17 & Reverse power through network protector & $\mathrm{X}$ & $\mathrm{X}$ & & & $\begin{array}{l}\text { What would be an acceptable ratio of the } \\
\text { minimum customer load current over the } \\
\text { maximum DR output to eliminate any possibility of } \\
\text { reverse power through a protector? }\end{array}$ & \\
\hline 18 & $\begin{array}{l}\text { Reverse power through network protector } \\
\text { (Note: This is really a subset of } 4 \text {.) }\end{array}$ & $\mathrm{X}$ & $\mathrm{X}$ & & & $\begin{array}{l}\text { What action needs to be taken with a sudden loss } \\
\text { of large load? }\end{array}$ & \\
\hline 19 & Reverse power through network protector & $\mathrm{X}$ & $\mathrm{X}$ & & $\mathrm{X}$ & $\begin{array}{l}\text { Can power swings or loss of synchronism by } \\
\text { rotating generators cause reverse power through } \\
\text { a network protector? }\end{array}$ & \\
\hline 20 & Unintentional islanding within the network & $\mathrm{X}$ & $\mathrm{X}$ & & $\mathrm{X}$ & $\begin{array}{l}\text { If the DR islands, how will the master relay be } \\
\text { prevented from reclosing the protector switch } \\
\text { during an out-of-synchronism condition? }\end{array}$ & \\
\hline
\end{tabular}


The following list of categories and subcategories was refined at the workshop:

Network Design/Settings

- Size, type, number of network units, voltage

- Network protector settings

- Automation

- Maintenance

Network Protector Unwanted/Undesired/Spurious Trip

- Reverse power

- Fault

Network Protector Operation

- Cycling

- Closing - PG\&E solution/ConEd box for $>50 \%$ of network protector online requirement (1547)

DR Design and Operation

- Normal conditions

- Fault conditions

- Coordination (settings, communications)

- Security

Islanding

For each subcategory, it will be necessary to develop specific issues, what major conditions apply, what solutions and needs are believed to exist, and priority. 


\section{References}

[1] ANSI C57.12.40-2000, ANSI Requirements for Secondary Network Transformers Subway and Vault Types (Liquid Immersed).

[2] IEEE Standard C57.12.44-2000, IEEE Standard Requirements for Network Protectors.

[3] IEEE Standard C37.108-1994, IEEE Guide for the Protection of Network Transformers.

[4] IEEE Standard 1547-2003, IEEE Standard for Interconnecting Distributed Resources With Electric Power Systems.

[5] California Public Utilities Commission. Electrical Rule 21, Generating Facility Interconnections.

[6] "Spot Network Equipment." Eaton/Cutler-Hammer Document \#0453, January 2003.

[7] Settembrini, R.C.; Fisher, J.R.; Hudak, N.E. "Reliability and Quality Comparisons of Electric Power Distribution Systems." IEEE Power Engineering Society, Transmission and Distribution Conference, 1991. 


\section{Appendix: Network Protector Ratings}

IEEE Standard C57.12.44-2000 establishes minimum requirements for network protector continuous current, interrupting current, and closing and latching current, as shown in tables A-1 and A-2. The close and latch ratings apply only to network protectors that have springclose or stored energy mechanisms. Network protector ratings exceed transformer nameplate current ratings so short-term overload capability of the transformer may be exploited.

Table A-1. IEEE Network Protector Ratings at 216/125 V

\begin{tabular}{|c|c|c|c|c|c|}
\hline \multicolumn{3}{|c|}{ Network Protector } & \multicolumn{3}{|c|}{ Network Transformer } \\
\hline $\begin{array}{c}\text { Continuous } \\
\text { Current } \\
\text { Rating } \\
\left(A_{\text {rms }}\right)\end{array}$ & $\begin{array}{c}\text { Interrupting } \\
\text { Current } \\
\text { (A } A_{\text {rms }} \\
\text { symmetrical) }\end{array}$ & $\begin{array}{c}\text { Close and } \\
\text { Latch } \\
\text { Current } \\
\left(A_{\text {rms }}\right. \\
\text { symmetrical) }\end{array}$ & $\begin{array}{c}\text { Nameplate } \\
\text { Rating } \\
\text { (kVA) }\end{array}$ & $\begin{array}{c}\text { Nameplate } \\
\text { Current } \\
\left(\mathrm{A}_{\mathrm{rms}}\right)\end{array}$ & $\begin{array}{c}\text { Protector } \\
\text { Rating } \\
(\% \\
\text { transformer } \\
\text { nameplate) }\end{array}$ \\
\hline 800 & 30,000 & 25,000 & 225 & 600 & 133 \\
\hline 1200 & 30,000 & 25,000 & 300 & 800 & 150 \\
\hline 1600 & 30,000 & 25,000 & 500 & 1333 & 120 \\
\hline 1875 & 30,000 & 25,000 & 500 & 1333 & 141 \\
\hline 2000 & 35,000 & 35,000 & 500 & 1333 & 150 \\
\hline 2250 & 35,000 & 35,000 & 500 & 1333 & 169 \\
\hline 2500 & 60,000 & 40,000 & 750 & 2000 & 125 \\
\hline 2825 & 60,000 & 40,000 & 750 & 2000 & 141 \\
\hline 3000 & 60,000 & 40,000 & 1000 & 2667 & 112 \\
\hline 3500 & 60,000 & 40,000 & 1000 & 2667 & 131 \\
\hline 4500 & 60,000 & 40,000 & 1000 & 2667 & 169 \\
\hline
\end{tabular}


Table A-2. IEEE Network Protector Ratings at $480 / 277$ V

\begin{tabular}{|c|c|c|c|c|c|}
\hline \multicolumn{3}{|c|}{ Network Protector } & \multicolumn{3}{|c|}{ Network Transformer } \\
\hline $\begin{array}{c}\text { Continuous } \\
\text { Current } \\
\text { Rating } \\
\left(A_{\text {rms }}\right)\end{array}$ & $\begin{array}{c}\text { Interrupting } \\
\text { Current } \\
\text { ( } \mathbf{A}_{\text {rms }} \\
\text { symmetrical) }\end{array}$ & $\begin{array}{c}\text { Close and } \\
\text { Latch } \\
\text { Current } \\
\left(A_{\text {rms }}\right. \\
\text { symmetrical) }\end{array}$ & $\begin{array}{c}\text { Nameplate } \\
\text { Rating } \\
\text { (kVA) }\end{array}$ & $\begin{array}{c}\text { Nameplate } \\
\text { Current } \\
\left(A_{\text {rms }}\right)\end{array}$ & $\begin{array}{c}\text { Protector } \\
\text { Rating } \\
(\% \\
\text { transformer } \\
\text { nameplate) }\end{array}$ \\
\hline 800 & 30,000 & 25,000 & 225 & 600 & 133 \\
\hline 1200 & 30,000 & 25,000 & 750 & 900 & 133 \\
\hline 1600 & 30,000 & 25,000 & 1000 & 1200 & 133 \\
\hline 1875 & 30,000 & 25,000 & 1000 & 1200 & 156 \\
\hline 2000 & 35,000 & 35,000 & 1000 & 1200 & 167 \\
\hline 2250 & 35,000 & 35,000 & 1000 & 1200 & 188 \\
\hline 2500 & 45,000 & 40,000 & 1500 & 1800 & 139 \\
\hline 2825 & 45,000 & 40,000 & 1500 & 1800 & 157 \\
\hline 3000 & 45,000 & 40,000 & 2000 & 2400 & 125 \\
\hline 3500 & 45,000 & 40,000 & 2000 & 2400 & 146 \\
\hline 4500 & 60,000 & 40,000 & 2500 & 3000 & 150 \\
\hline 5000 & 60,000 & 40,000 & 2500 & 3000 & 167 \\
\hline
\end{tabular}




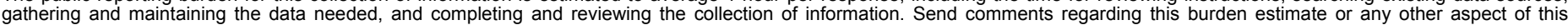

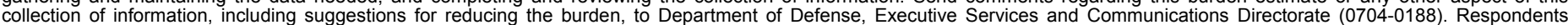

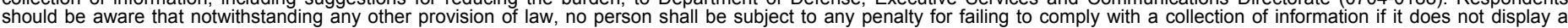

should be aware that notwithstanding

PLEASE DO NOT RETURN YOUR FORM TO THE ABOVE ORGANIZATION.

\section{REPORT DATE (DD-MM-YYYY) July 2005}

4. TITLE AND SUBTITLE

Secondary Network Distribution Systems Background and Issues

Related to the Interconnection of Distributed Resources
3. DATES COVERED (From - To)

5a. CONTRACT NUMBER

DE-AC36-99-G010337

5b. GRANT NUMBER

5c. PROGRAM ELEMENT NUMBER

5d. PROJECT NUMBER

NREL/TP-560-38079

5e. TASK NUMBER

DP05.1001

5f. WORK UNIT NUMBER
7. PERFORMING ORGANIZATION NAME(S) AND ADDRESS(ES)

National Renewable Energy Laboratory

1617 Cole Blvd.

Golden, CO 80401-3393
8. PERFORMING ORGANIZATION REPORT NUMBER

NREL/TP-560-38079

9. SPONSORING/MONITORING AGENCY NAME(S) AND ADDRESS(ES)

10. SPONSOR/MONITOR'S ACRONYM(S) NREL

11. SPONSORING/MONITORING AGENCY REPORT NUMBER

12. DISTRIBUTION AVAILABILITY STATEMENT

National Technical Information Service

U.S. Department of Commerce

5285 Port Royal Road

Springfield, VA 22161

13. SUPPLEMENTARY NOTES

14. ABSTRACT (Maximum 200 Words)

This document addresses the technical considerations associated with the interconnection of distributed resources (DR) with secondary network distribution systems. It provides an overview of the characteristics of distribution systems and interconnection requirements and identifies unique issues specific to network interconnections. It also identifies the network-specific interconnection issues for which test protocols should be developed. Recommended criteria and requirements for the interconnection of DR with network distribution systems are presented.

\section{SUBJECT TERMS}

distributed resources; DR; distributed energy resources; DER; interconnection; network distribution; distribution systems; standards; IEEE 1547; National Renewable Energy Laboratory; NREL

\begin{tabular}{|c|c|c|c|c|}
\hline \multicolumn{3}{|c|}{ 16. SECURITY CLASSIFICATION OF: } & \multirow{2}{*}{$\begin{array}{l}\text { 17. LIMITATION } \\
\text { OF ABSTRACT } \\
\text { UL }\end{array}$} & \multirow{2}{*}{$\begin{array}{ll}\text { 18. } & \text { NUMBER } \\
\text { OF PAGES }\end{array}$} \\
\hline $\begin{array}{l}\text { a. REPORT } \\
\text { Unclassified }\end{array}$ & $\begin{array}{l}\text { b. ABSTRACT } \\
\text { Unclassified }\end{array}$ & $\begin{array}{l}\text { c. THIS PAGE } \\
\text { Unclassified }\end{array}$ & & \\
\hline
\end{tabular}

19a. NAME OF RESPONSIBLE PERSON

19b. TELEPHONE NUMBER (Include area code) 